\title{
The Options Are Available for Controlling Residual Risk
}

\author{
Zitong Gao* \\ Jinshan Vocational Technical College, Yangzhong 212200, Jiangsu Province, China
}

\begin{abstract}
Macquarie Dictionary define 'Risk' as the chance of an adverse event depending on the circumstances. In the field of engineering, the risk is often described as a specific unwanted event and including the consequences or loss. Usually residual risk from civil engineering project would lead to serious effects. The control of residual risk is an important subject in modern civil engineering management research. In this paper, a residual risk controlling methods will be present. Firstly, the definition and other mentioned concepts will be introduced. It includes the definition of residual risk and other concepts of risk that are often confused. Next, basic ways of dealing risk will be mentioned.
\end{abstract}

Key words: Risk management; Residual risk; Secondary risk

Publication date: May, 2021; Publication online: 31 May, 2021

*Corresponding author: Zitong Gao, 2531244473@qq.com

\section{Definition about Residual Risk:}

The residual risk in this paper only refers to the 'residual risk' in the engineering project, which is not the same concept as the residual risk in other industries. According to information security management requirements ISO27001, residual risk is "the risk remaining after risk treatment". ISO/IEC27001 was proposed by the British Standards Institute (BSI) in February 1995 and revised in May 1995. The strength areas for BSI are health, electrical, engineering, materials, chemicals, consumer goods and services, information technology. BSI has thus become one of the world's recognized standards in the field of engineering.

Residual risk is the risk that remains after nearly all efforts to eliminate, the risk still exist. This also can be defined as the modified risk has been implemented; however, it could still affect results. Usually in actual projects, residual risks should be further evaluated and sorted out. Generally speaking, residual risk is after considering all external elements, the risk will remain at a certain level. This means this kind of risk is 'unavoidable'.

\section{Differences between Residual Risk and Inherent Risk:}

Residual risk is a different concept from inherent risk, and often beginner or less experienced project managers may confuse the two concepts. Inherent risk is the risk level before management actions, which is generally composed of contractual reasons and technical reasons. The most important difference between these two risk is residual risk be controlled in the whole project schedule, but inherent risk is that an activity would pose no controls factors. It has been mentioned in the last section that residual risk is inevitable, but this does not mean that it is an uncontrollable factor.

Why we need to compare these two risks is that they are usually assessed in the same way. This is why they are often lumped together. The Institute of Project Managers (APM) published a systematic approach to risk management in 1997, which is called Project Risk Analysis and Management (PRAM), and it defines a set of risk management steps. The main elements include specification, focus, identification, framing, ownership, estimation, evaluation, layout, and control. In addition, the process is simplified as organization and scope, identification, risk analysis, risk solution, response planning, continuous management and feedback.

Residual risk is tending to define within the simple risk model; however, the inherent risk is more problematic. The risk cannot be eliminated completely and control the risk to an acceptable level is our target. 


\section{Differences between Residual Risk and Secondary Risk}

The PMBOK® Guide defines secondary risks as "those risks that arise as a direct outcome of implementing a risk response". In simple ways, managers already identify a risk and have a response plan to deal with that one. Image this plan had been successful implemented, the new risk could arise from this implementation, which is called a secondary risk. Differently, residual risks are the leftover risks. The secondary risk is required actions and it takes creation of a response plan. For residual risk, it not always needs actions and depends on the situations. It may need a contingency action plan.

For instance, in order to have a road construction, the engineers decided to close George St. This means cars cannot go through George St; however, a bicycle may get into the construction site. This kind of risk is called secondary risk.

Why we need to compare these two risks is that they are usually being confused. Engineers may have established a risk of traffic congestion that may last 10 days or one month and which may not disrupt the construction. To manage this risk, engineers have scheduled other plan with a buffer of couple of days. So that even if it traffic congestion for few days, other plans are not disrupted. Even though it has a risk control, this does not eliminate the risk of the timetable getting messed up; but only lowers the risk. The risk still exists, which is called 'residual risk'.

\section{Negative result from residual risk:}

- Adverse result of operation (e.g. decrease of productivity);

- Adverse result of management (e.g. high human resource turnover rate);

- Adverse result of budget (e.g. high cost about maintenance of equipment);

- Adverse result of corporate reputation (e.g. project delay);

- Adverse result of personal safety (e.g. fire)

\section{Basic ways of dealing risk:}

The control methods will help reduce the damage in the effects of risk but it cannot remove the danger completely. What remains there after taking all these controls into account is a residual risk.

\section{Appetites for risk}

The project managers, evaluate the adequacy and effectiveness of these controls in order reduces residual risk to an acceptable level by the board. If the acceptable level of risk is higher than the level of risks, the manager could accept these risks formally.

\section{Reassess risk plans}

If the acceptable level of risk is below than the level of risks, there is a requirement about evaluation, reviewing and analysis of plan implementation.

Risk Priority Number (RPN) without Control = Frequency * Detection * Severity (1.1)

Residual Risks with Administrative Control $=$ Frequency

* Detection * Severity (1.2)

Risk Rating (RR) = Frequency (F) $x$ Likelihood (L) $x$ Severity (S) (1.3)

3. Reconsider the Construction Project Feasibility Studies

If all feasible and practical actions have been widely undertaken to decrease the risks but still has high risks, a system of residual risk approval should be developed and applied.

\section{Disclosure statement}

The author declares no conflict of interest.

\section{References}

[1] Bignozzi, V. and Tsanakas, A. (2015). Parameter Uncertainty and Residual Estimation Risk. Journal of Risk and Insurance, 83(4), pp.949-978.

[2] Ishandbook.bsewall.com. (2017). Inherent \& Residual Risk.

Available

at:

http://ishandbook.bsewall.com/risk/Assess/Risk/inherent_ris k.html [Accessed 10 May 2017].

[3] Park, Y. (2016). Project Management Using a Risk Mode and Action Plan Method. The Korean Academic Association of Business Administration, 29(10), pp.1549-1564.

[4] Leigh, N (2017). What is Residual Risk? - Objective Controls. Available at:http://www.objectivecontrols.com/blog_2015/2015-08-12 _objective_blog.html [Accessed 10 May 2017]. 